\title{
COMMENTS ON SOME MINOR SURGICAL CONDITIONS
}

\author{
By D. Lang Stevenson, F.R.C.S.E. Late Lieut.-Col., R.A.M.C. \\ Surgeon, Whipps Cross Hospital
}

Pilonidal sinus, phimosis, hydrocele, varicose veins and anal complaints are familiar in the list of conditions which commonly inflict varying degrees of discomfort in the younger age group of the population. Implicit in the undertaking of treatment is the expectation that comfort or cure can be achieved by a relatively minor procedure followed by a rapid convalescence and return to work. The most mutually satisfying extent to which this expectation may be realized is conditioned in the majority of cases by the method of surgical technique and management. True, one method may differ from another only in detail, but it is the detail that may in large measure decide the smoothness of the post-operative course, the quality of the end result, and the time spent in hospital or off work. In these respects the subsequent modifications and details of technique have proved valuable in the treatment of the conditions already mentioned. Some comments have also been made on evaluation of treatment particularly in regard to varicose veins.

\section{Pilonidal Sinus}

The difference between primary and secondary healing in these cases almost implies the difference between three weeks and three months in hospital. The essentials for success in primary suture are well recognized in complete excision, meticulous asepsis, and a method of closure which eliminates dead space. The last is of first importance since most failures are initiated by tenting of the superficially healed skin and subcutaneous tissues. The cardinal point is to ensure that the soft parts are not only in mutual apposition but that they are also in close contact with the aponeurotic covering of the sacrum. Indeed, the principles are the same as in the application of a full thickness skin graft to an awkward contour. The two factors which militate against success are the relatively avascular sacral bed and the forces of tension.which are constantly persuading the sutured soft parts to assume the more relaxed position away from the sacrum. For these reasons a pressure dressing is a sine qua non and must be maintained for at least 14 days. If removed too soon, the skin and subcutaneous tissues tend to spring away from the sacrum at the first movement which increases local tensions. Sero-sanguinous exudate is the natural reaction, and superadded infection is not far behind. This probably accounts for a number of disappointing results in cases which seemed at first to be doing well, and also indirectly for the multiplicity of techniques now advocated.

All active infection must have settled before operation is contemplated. Incision and drainage of an abscess may be a necessary first phase in treatment and the opportunity should be taken of curetting away gently as much debris as possible. A very fine probe may establish to which side of the mid line the main sinus deviates. The skin is prepared for three days before operation and the bowels for six days confinement. At operation the skin of the whole area is thoroughly treated with a suitable antiseptic and the anus is sealed off with a응 dressing. The orifice of the sinus is given an application of pure carbolic acid and later obliterated by approximating the skin edges resulting from the eliptical incision.

After excision of the sinus an anchor type of pressure dressing is essential, and is the keystone of success in this operation. Although this form of dressing has been described and used in many ways, an outline of the following modification is justified by the end results. Three or four pairs of specially placed deep sutures of strongest nylon are distributed over the length of the wound and the ends of each pair are secured with forceps. With a large curved needle the suture commences one inch from the skin edge at the upper extremity of the wound, passes through the full thickness of the soft parts, takes a good bite of aponeurosis just to the same side of the mid-line, and returns through the full thickness of the soft parts again emerging about three-quarters of an inch below the starting point. Three or four such sutures are so placed that pairs do not cross in the mid-line (see diagram). The skin edges are brought together with fine interrupted vertical mattress sutures which are tied lightly. It is well to emphasize here that the minimum of skin should be sacrificed at the excision of the sinus. Before inserting the final stitch any blood that may have collected in the wound is expressed. The anchor 

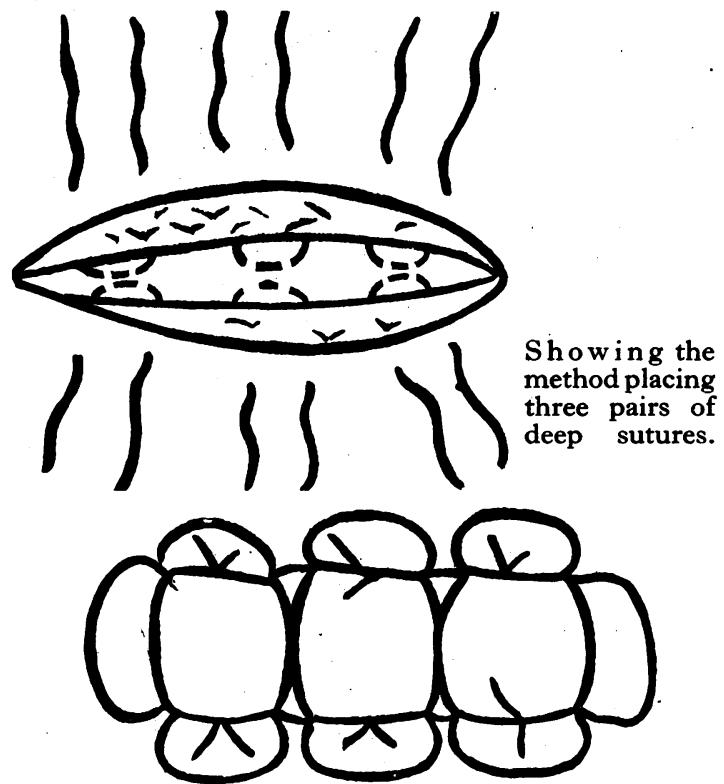

surgical treatment in terms of hospital days can be justified in relation to the minor nature of the disability.

\section{Phimosis}

An oedematous and ulcerated suture line may keep a man in hospital or off work for several weeks quite apart from the alarm such an .appearance may invoke in the patient. Certain points in this operation must be observed if these complications are to be avoided. The areolar tissue in this region, like that of the eyelid, quickly reacts with oedema to the most trivial irritant. The object of the following technique is to avoid all sources of tissue irritation as far as possible. Forcipressure and torsion with a fine type of mosquito forceps is all that is used and all that is necessary for haemostasis. Buried catgut should never be employed. Nylon as a suture material has few equals judged by the kindly way it is tolerated by all tissues and experimental work endorses this experience.

It is hoped that the diagram demonstrates the

The completed Anchor dressing. A separate sterile dressing is tucked loosely under the anal end of the long gauze roll.

Fig. I

dressing (which consists of one long and three shorter rolls) is now applied. The large roll is placed along the wound and the three shorter rolls are laid across it in such a manner that each pair of deep sutures may be tied over the corresponding extremity. These sutures are tied, first on one side and then with the short rolls drawn tightly across the long one, the remaining sutures are knotted (see diagram). It is important to tighten the nylon slowly feeling for the optimum tension to make the knot. Finally a sterile dressing is tucked under the lower end of the long roll.

The secret lies in making the anchor dressing of generous size and soft consistency and in tying the deep sutures at the right tension. Twice daily ether is run over the dressing to maintain dryness of the surrounding skin. The bowels are confined for six days and on each future occasion when the bowels have moved the sterile dressing under the lower end of the long roll is changed. Parenteral penicillin is given if indicated. When the anchor dressing is, removed on the 14 th day the wound is found to be soundly healed by first intention and all sutures are removed. The patient is able to leave hospital on the r6th day.

Many people would prefer to tolerate the nuisance of a slight discharge and an occasional abscess than endure three months of daily dressing while lying in an uncomfortable or restricted position. In fact simple incision, gentle curettage and penicillin may achieve quiescence for months. It is only by making primary healing the rule that method and how these requirements are satisfied. Fine nylon on a straight cutting needle is used to place the six necessary sutures. The first is at the fraenum and is the well-known corner stitch modified by wider spacing. The ends are left untied (see diagram). The next suture, placed at 12 o'clock, is returning in type and makes the loop on the corona. The ends again are left untied and secured with forceps. Two sutures are now inserted at approximately io o'clock and 2 o'clock, and these differ from the 12 o'clock suture only in that the loop is on the foreskin. The last pair are at 8 and $40^{\prime}$ 'clock, the loop being once more on the corona. All stitches must be accurately made close to the margin of the integument. Two gauze rolls are now prepared about as thick as the little finger and preferably sewn with a rapid basting stitch. The long roll is made to surround the corona like a collar and the short one is laid lengthwise on the fraenum. Before this is done each is smeared lightly with a little Vaseline on the narrow strip of gauze which is to lie in contact with the suture line. Firstly the short roll is placed on the fraenum and the nylon is gently tied over it leaving the ends long. When the large roll is placed in position the free ends of the sutures should lie on their respective and alternate sides (see diagram). The ends of the 12 o'clock suture are then tied to the adjacent ends of the ro and 2 o'clock sutures, while the two opposite ends are tied to the adjacent ends of the 4 and 8 o'clock sutures. Gentle traction on the two remaining ends. fixes the dressing in position and these are finally tied to the long ends of the suture at the fraenum. It will be seen that the nylon now takes a con- 


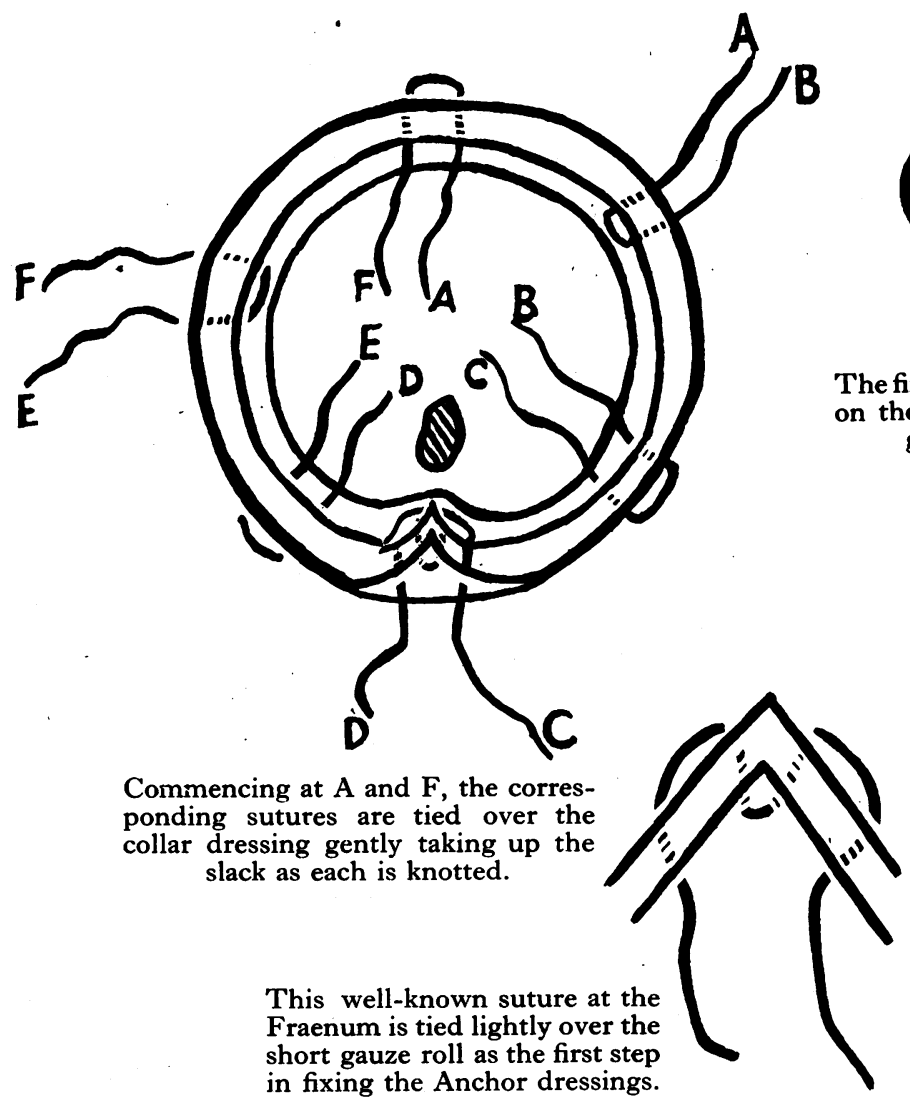

Fig. 2

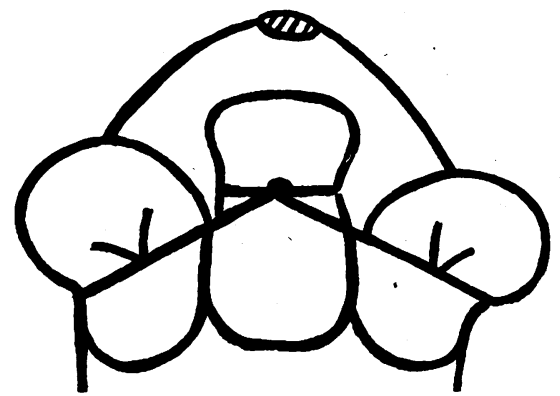

The final position of the sutures and Anchor dressings n the ventral aspect of the penis. Note the short gauze roll is laid proximal to the meatus.

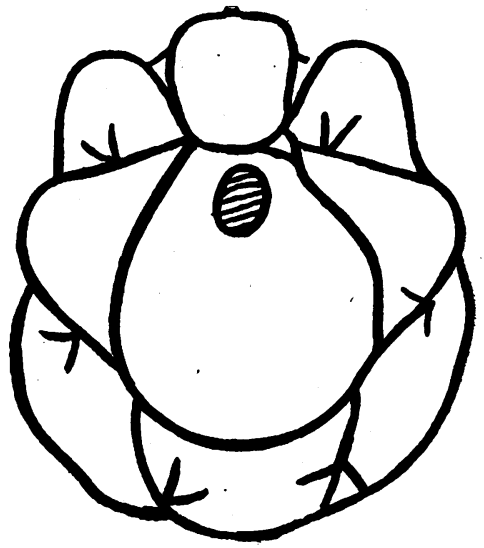

An 'end on' view of the completed dress ing. It is removed by cutting on eithe믐 side of each knot.

Fig. 3 tinuous spiral course over the gauze roll and obtains apposition of the skin edges by linear tension, no knot being tied on the skin. The gauze roll on the fraenum is trimmed to lie behind the meatus and the patient is instructed to dry the meatus after urination with a pledget of cotton wool. The suture is removed on the third day by cutting on either side of the knot over the gauze. The dressing falls off and the sutures slide out so easily that the patient is usually unaware of the fact.

Adult circumcision is an operation which demands more care and precision than the average surgical textbook seems to indicate. If the edges of the foreskin and corona are cut cleanly and all details in the above technique are observed, exact healing by first intention can be obtained in every case. The combined protective and pressure dressing has also the advantage that it is extremely comfortable, allowing the patient to be ambulant from the day of operation.

\section{Hydrocoele}

The difference between the uncomplicated operation for hydrocoele and the case which is complicated by an infected haematoma may be the difference between six days and six weeks in hospital, quite apart from the unpleasant end result. It is therefore of first importance that the operation is as far as possible avascular and that haemostasis is meticulous.

The following technique fulfills these requirements. Apart from minor modification it is a method which I learned from my former chief, Mr. E. T. C. Milligan. The scrotum is compressed and steadied with the left hand and an incision is made through the skin and dartos muscle, the length according to the size of the hydrocoele. (The belief that scrotal incisions have a tendency to become infected is without foundation and indeed these wounds heal with a practically invisible scar if care is taken to use an everting form of suture. Dislocating the scrotal contents into the inguinal region is a more traumatic and vascular procedure which should be condemned.) All fascial coverings are divided until the blue translucent parietal tunica vaginalis is exposed, which must be done with great care since the delicate membrane is easily ruptured. A combination of blunt and shàrp dissection com- 
pletes the mobilization of the cyst. If the closed limbs of a pair of non-toothed dissecting forceps are insinuated beneath a layer of tissue and allowed or compelled to open, the tissue is placed on tension and elevated from deeper structures : this manoeuvre greatly facilitates division of the tissue with scalpel or scissors and is of particular use in this operation. Incidentally, the correct use and application of tension is one of the great secrets of technical surgery. The tunica vaginalis is now divided down the middle and reflected behind the testicle. The edges are clipped together for two or three minutes with Kocher forceps which usually suffices to effect adherence. Any bleeding point must be ligated at sight although as a rule there are few or none at all. The testicle is now gently replaced by holding up the edges of the scrotum with four strategically positioned tissue forceps. The wound is closed without drainage by a few interrupted vertical mattress sutures of fine nylon. A sterile suspensory bandage lined with a layer of wool makes a satisfactory and comfortable dressing. The sutures are removed on the third day and the patient leaves hospital on the next day with a support.

This operation is effective in all simple uncomplicated hydrocoeles. Although it is alleged that these modified Jaboulay types of operation have the defect of occasional recurrence, the writer has not seen this. It is well to remember that many patients can get along quite comfortably with repeated tappings, but if operative cure is to be preferred to this form of treatment or injection therapy, it should be a simple procedure involving only a short period off work.

\section{Varicose Veins}

In modern practice the two chief indications for obliteration or ablation of varicose veins in the lower limbs are improvement of appearance and improvement of function. In the former, where treatment is almost restricted to women, the results, on the whole, attain the objective. In the latter the problem is neither as simple nor as satisfactory, and there are several factors which contribute to this state of affairs.

The too mechanistic approach, which identifies the ache with visible varicose veins, assumes that removal of the vein must in all cases remove the ache. This belief tends to overlook physiology, pathology, psychology, and even experience itself. It is common knowledge that some possessors of the most gross varicose veins have no trouble throughout an active lifetime and indeed may be able to walk 20 miles over rough country. This demonstrates that the size and extent of the veins bear no constant relationship to the disability and that there is, therefore, no true causal relationship.
On the assumption that symptomless varicose veins do not require treatment in the male, and that a cosmetic operation is rarely desired by a man, the only indication for treatment in this sex is disability. Since clinical assessment of the degree of disability depends in large measure on the patient's subjective evidence, there can be no guiding rule in selection which must remain a matter for individual judgement. However, unnecessary treatment can at least be avoided by recognizing that there are certain groups of men with symptomless varicose veins who may nevertheless be subjected to surgical procedures. The limitations of modern therapy must also be borne in mind. Firstly, there are those cases where the real disability lies in foot strain, pes planus or other orthopaedic defect. Secondly, there are those who seek treatment because they believe that varicose veins, like certain dread diseases, must be caught early to be cured, and not because the veins are giving trouble. This can be understood since the unpleasant complications in advanced cases are widely known to the public but in most instances convincing reassurance relieves the apprehension and the veins are forgotten. The third group comprise candidates for various services, foremost among which is the Police Force. Here, treatment before acceptance is obligatory. This means that a young man of I8 or I9 with an early incompetency of the saphenous system is compelled to undergo an operation for ablation or obliteration of the veins. This policy is not in the interests of long and efficient service as his veins might be symptomless for another ten years or never trouble him at all. On the other hand an untimely operation may take him off his beat in half that time by antedating the onset of a disability or even by causing an avoidable one. It would seem that at some point this policy has been endorsed by the medical profession.

Since varicose veins are in most cases familial and have a tendency to increase in size and extent throughout life, treatment, at the best, merely modifies the effects of the tendency by improvement in appearance or function for a varying period of time. How long the improvement is maintained depends, of course, on the presence of favourable or unfavourable factors. In the latter group long standing occupations and pregnancy are the first to be indicted. Conversely, a sedentary job is one of the best guarantees of a reasonably lasting result, and indeed all the treatment that some cases require. Moreover, there appears to be a limit in the extent to which the superficial venous system of a varicose subject may be removed by repeated obliterations and excisions without jeopardizing muscular circula- 
tion and function. A limb that has been denuded of the varicose saphenous systems may show little evidence of visible varicosities yet a bursting pain is experienced on slight exercise. This condition might well be called varicose veins sans varicose veins. Any major ablation must throw an increased strain on both deep veins and the remaining superficial veins. The latter being unsupported by muscle and suffering from the same inherent structural defect gradually become varicose. Taking the mechanistic approach to its logical conclusion, the belief that repeated removal of these further varicose veins as they appear will maintain normal function cannot be reconciled with experience.

Treatment should, therefore, be planned in regard to the active life span of the individual so that the maximum benefit accrues at the optimum period. Also, judicious selection of the patient and the treatment for the patient are of equal importance. The writer now reserves injection of sclerosing agents mainly for cosmetic treatment in women. Where disability exists, excision of the veins gives better results and the possibility of deep thrombosis is greatly diminished if not eliminated. Operative treatment is avoided in men below the age of 30 , which incidentally is also a good rule in the case of other varicose conditions. The case with a single large localized group of veins in the calf is the type of case which seems to benefit the most from excision. The extensive incompetency of the great saphenous system with disability is treated, in the first place, by simple ligation at groin and knee. Two or three months later the case is reviewed since a number of these cases are improved quite appreciably by ligation alone. If indicated, excision can then be undertaken. Lastly, in suitable cases considerable use is made of a well-made elastic stocking both as an adjuvant or alternative to surgical treatment.

Wound infection is generally ascribed to faulty asepsis, haematomata, or ' dead space.' A possible explanation of the characteristic sullen induration which may keep a man in bed several weeks, is damage to infected lymphatics at dissection. At least where simple ligation is performed such cases are rarely encountered.

Some points in regard to technique may be worth mentioning. The removal of a length of vein may be easily performed with the writer's ball-ended needle originally designed for synchronous injection (Lancet, Jan., 1938). The ball end is passed down the vein as far as possible and a small incision is made over it : when the vein has been exposed a ligature is tied above and below the ball. The vein is divided between the ligatures and by withdrawing the needle forcibly, the vein is turned inside out as it follows the needle.
Further segments of vein can be removed in the same way through small incisions. A new method of obliterating varicose veins in continuity is at present being tried out by means of a ball-ended electrode and a further report will be made at a later date since the results so far are most promising. The technique has the advantage that a certain sclerosis is obtained with the minimum of intraluminal altered blood and without the risk of the irritating agent entering the deep veins, which is one of the disadvantages of injection therapy.

To sum up, the problem of selection is of equal importance with the matter of technique. In the male at any rate where cosmetic considerations are not important, the criterion for treatment must be the maintenance of optimum function throughout the active life span. In this context the concluding aphorism is perhaps apposite small veins with symptoms not yet, large veins without symptoms never.

\section{Anal Complaints}

Occasional bleeding at defaecation is a not uncommon complaint in a young man and as a rule there is no call for treatment other than liquid paraffin and local ointment for a few days. The single thrombosed external haemorrhoid is another familiar condition at this age which is treated according to size and pain, conservatively or by evacuation of the clot. The cause is probably to bece found in the common systemic venous pool which drains both the genital and anal regions, congestion in the former being shared by the latter. The writer avoids operating on internal haemorrhoids below the age of 35 if possible. Many patients with moderate internal haemorrhoids can be made comfortable by correcting faulty habits and by instruction in defaecation. Quite large prolapsing internal haemorrhoids can be treated successfully by injection although it is preferable to admit these patients to hospital for two days. The bowel is prepared as for haemorrhoidectomy and a large injection of ro to $15 \mathrm{ml}$. 5 per cent. phenol in oil is given. For large haemorrhoids this régime has been found to be more satisfactory than multiple serial injections. Should the haemorrhoids prolapse during the first ten days, the patient is instructed to replace them digitally.

Acute, and even in some cases chronic, fissure in ano may often be healed by correcting the bowel action and by local baths and ointment. A further valuable adjunct to treatment is to instruct the patient to carry out digital dilation in a hot bath with soapy water, which, incidentally, is a good practice after pile operations. In the matter of correcting causes, it is interesting to observe that not infrequently emotional conflict or disturbance takes first place in the chain of aetiological factors. 
For chronic fissure in ano, surgical excision and suture has been practised with surprisingly good results. A stay suture is inserted at the proximal and distal extremities of the fissure which is made prominent by tension on each stay. The fissure is then easily excised with the subjacent portion of the subcutaneous external sphincter. Two or three figure-of-eight sutures of fine nylon are placed, one of which approximates the cut ends of the sphincter. A layer of gauze is wrapped round a small soft rubber tube and the sutures are tied lightly over it. The bowels are confined for six days and the dressing remains clean until the sixth day when the sutures are removed and an olive oil enema is given. The sutures are easily removed by cutting over the dressing. Liquid paraffin by mouth and local. Vaseline before defaecation are used for two subsequent weeks, and it is interesting to note that the writer has not seen fistula formation after this procedure. There is also an economy in hospital days as compared with wounds allowed to heal by second intention.

Time lost from work in the treatment of a minor disability, although of secondary significance to successful treatment, is nevertheless of major importance to the individual and to the community. The aim of the modifications in technique and management which have been described, has been to achieve cure or comfort in the shortest possible time. The guiding principle has been close attention to the smaller clinical and technical details.

\title{
TREATMENT OF ACUTE EMPYEMA THORACIS WITH INTRAMUSCULAR AND INTRAPLEURAL PENICILLIN
}

\author{
By W. A. Elliotr, M.D. (Camb.), M.R.C.P. \\ Trainee Specialist Physician \\ and \\ $\Varangle \quad$ B. A. Young, M.D.(Lond.), M.R.C.P. \\ Physician Superintendent, St Alfege's Hospital, Greenwich
}

This paper is based on a series of 22 cases of acute empyema admitted to this hospital between March, 1945, and Auguist, 1947. The underlying disease was lobar pneumonia in 18 cases, bronchopneumonia in one case, lung abscess in one case and neoplasm in two cases. The duration of symptoms before admission to hospital varied from three days to six weeks. (The onset of symptoms is reckoned from the first day on which the patient felt ill, as the date of onset of the empyema cannot be accurately determined.)

While acute toxaemic symptoms were present, the patients were given intramuscular penicillin in doses sufficient to maintain a continuous bacteriostatic concentration of the antibiotic in the blood-stream (usually 60,000 units four-hourly). In addition, the pleural cavity was aspirated as completely as possible through a wide bore needle on alternate days and intrapleural penicillin (usually 250,000 units) was injected. (Smaller doses were given in some of the earlier cases in the series.) The fluid withdrawn was cultured and the sensitivity to penicillin of the organisms grown, was estimated. Breathing exercises were started as early as possible in all cases.
This method of treatment gave good results in I 4 of the pneumonic cases and in the lung abscess case. Details are given in the table below. The average time spent in hospital in these cases was 52 days; the average time after this for the chest radiographs to become clear was five weeks. Good functional results were obtained in all 15 cases. In four other pneumonic cases, rib resection and drainage was carried out after a course of intramuscular and intrapleural penicillin. In one of these four cases, the culture of the pus from the pleural cavity grew bacterium coli which was resistant to penicillin. In another case, in which

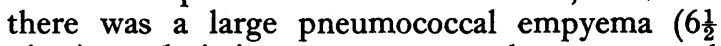
pints) on admission, a pyo-pneumothorax occurred during treatment. The remaining two cases which were treated surgically, were early in the series and received relatively small doses of penicillin ; one of them having no intramuscular penicillin. In the light of our later experience we consider that both these cases would probably have cleared up without operation had larger doses of penicillin been given. The remaining pneumonic case improved considerably with intramuscular and intrapleural penicillin and the patient was dis- 